\title{
Inverse Problem of Paleomagnetic Reconstruction: Formulation
}

\author{
Masaru KONO* and Hideo UCHIMURA \\ Department of Earth and Planetary Sciences, Tokyo Institute of Technology, \\ Ookayama 2-12-1, Meguro-ku, Tokyo 152, Japan
}

(Received February 22, 1993; Revised January 25, 1994; Accepted January 25, 1994)

\begin{abstract}
Paleomagnetism provides data useful for reconstructing the former positions of the continents. Conventionally, reconstructions were performed by constraining the continental blocks to latitude circles defined by paleomagnetic data, while the longitudinal positions were chosen arbitrarily. We propose a new method of reconstruction based on the geophysical inverse theory, in which models are evaluated by the combined effects of misfit and penalty, the latter being given by some physical reasoning. We allow misfits in reconstruction within the uncertainties given by the statistical parameters of paleomagnetic data. For the penalty function, we propose to minimize the total slip of blocks accompanied with the movements over the surface of the earth. The method is formulated and then applied to the simple two-plate problem of the opening of the North Atlantic. Although the reconstruction looks reasonable, there is certain discrepancy between the obtained model and the Bullard et al. fit of Europe and North America. The reason for this discrepancy as well as other practical problems in obtaining the reconstructions are discussed.
\end{abstract}

\section{Introduction}

Paleomagnetism provides a very important source of data for the studies of global and regional tectonic history. It is well known that paleomagnetic data and its interpretation played a key role in reviving the continental drift hypothesis of Wegener (1929) and preparing for the development of plate tectonic theory (e.g., Uyeda, 1978). An important feature of paleomagnetic data is that they are essentially quantitative; if the dipole hypothesis is correct and if the secular variation is appropriately averaged out, they directly give the paleolatitude and so define the position of the pole at that time. This procedure of mapping paleomagnetic data into poles contains errors, but the errors themselves can again be treated in a quantitative way through Fisher's (1953) or other statistical methods. It is this quantitativeness which distinguishes paleomagnetic data from other more or less qualitative data used in tectonic studies.

Following the establishment of plate tectonics and especially the validation of Vine and Matthews (1963) theory, the role of the most quantitative data used in the reconstruction of the movement of the blocks in the past has been taken over by oceanic magnetic anomalies, which are again paleomagnetic data in a broader sense. However, the time interval for which magnetic anomalies are usable for reconstruction is at most the last $200 \mathrm{Ma}$, which is a small portion of the earth's tectonic history. Before the middle Mesozoic, paleomagnetic data is still the only quantitative data available to us.

Paleomagnetic data, however, are far from ideal. They permit substantially different interpretations which satisfy the observational data equally well. This nonuniqueness in interpretation arises from two different sources. Firstly, "continental drift" and "polar wander" are complementary concepts in the sense that paleomagnetic data from a single block can be interpreted by

*Present address: Department of Earth and Planetary Physics, University of Tokyo, Yayoi 2-11-16, Bunkyo-ku, Tokyo 113, Japan. 
one or the other or their combination, and their contributions cannot be separately determined. Secondly, even when the true polar wander is known precisely, paleomagnetic data from a block determines its distance from the pole ("magnetic colatitude") as well as its orientation with respect to the cord drawn from the pole ("declination"), but they do not constrain its "longitude".

An example of such nonuniqueness is illustrated in Fig. 1 for the case of a single block and its associated apparent polar wander path (APWP). At one extreme, it is possible to interpret the data in such a way that the block never moved from its present position and only the pole moved as shown by the APWP (Fig. 1a). This interpretation completely satisfies the data at hand. It is also possible, however, to interpret this data set by a pole fixed to the present north pole and movements of the block along a meridian (Fig. 1b). A variant of the second interpretation is that the block moved not only in the meridional direction but in longitudinal direction as well (Fig. 1c). The above example shows the possibility of interpreting the same paleomagnetic data by polar wander only (Fig. 1a), by continental drift in latitudinal direction only (Fig. 1b), or by drift in both latitudinal and longitudinal directions (Fig. 1c). Also to be noted is that there is a case in which the block stayed at the same position, yet changed its orientation in reference to the present coordinate system (Fig. 1d).

Tectonic reconstruction is basically a problem of finding relative movements between multiple blocks, so that the interpretation of an APWP from a single block does not constitute a realistic problem. Moreover, for two adjacent continents, it is often observed that the range of possible "reasonable" interpretation is much reduced from practical considerations (e.g., Irving et al., 1974). However, the fundamental fact remains that the determination of tectonic movements by
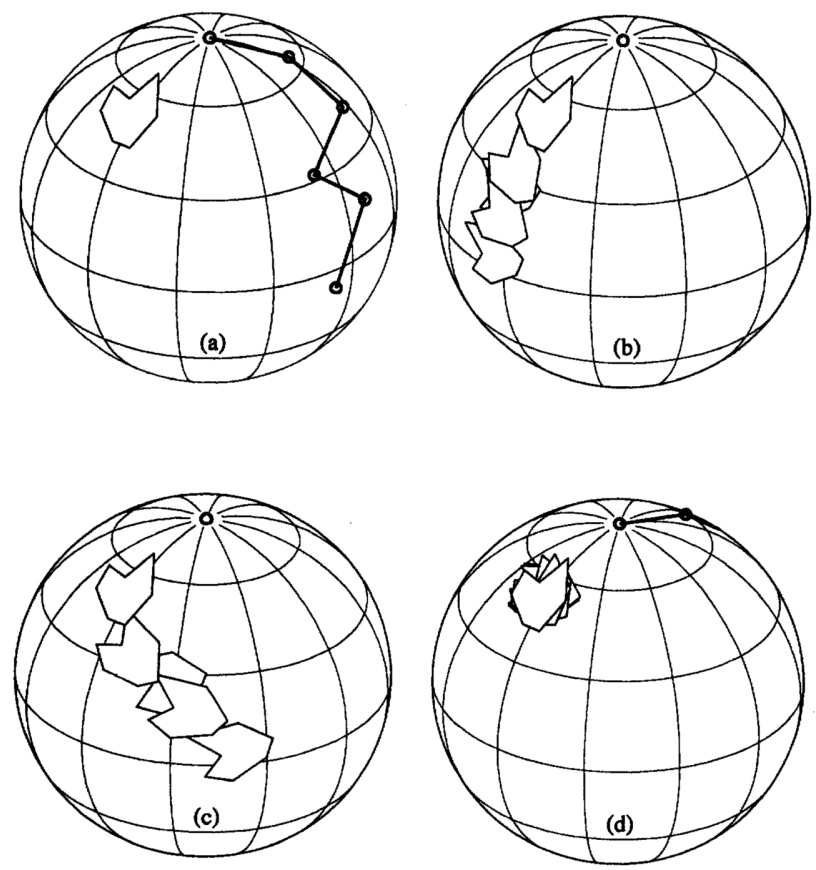

Fig. 1. Some of the possible interpretations of an apparent polar wander path (APWP) for a single block. Note that all the interpretations equally satisfies the data. (a) If the block never moved, the APWP shows the true polar wander path. (b) The pole remained fixed all the time, and the block moved along the meridian changing its orientation. (c) As in (b), but the block moved not only in north-south but also in east-west direction. (d) The block stayed at the same position but rotated (polar wander remains in this case). 
paleomagnetic data is always a nonunique process.

Backus and Gilbert (1967) pointed out that, in any geophysically plausible situation, observational data can almost invariably be fitted by infinitely many models. They showed that from this manifold of models the most reasonable one can be chosen by minimizing a combination of the misfit and a regularization function obtained from physical reasoning. Jackson (1972) formulated the inverse problem when the functions are given at discrete values of independent variables. These treatments show that nonuniqueness in defining a model is not restricted to paleomagnetic data but is a fundamental feature of any geophysical data set. Conversely, paleomagnetic reconstruction may be formulated in the way of the general geophysical inverse problems which enable us to estimate the nonuniqueness involved in the model. This paper is a report of such a trial. In the following we will present a scheme to formulate paleomagnetic reconstructions as an inverse problem.

\section{Formulation as an Inverse Problem}

In paleomagnetism, observational data are the inclination $I$ and declination $D$ representing the magnetic field direction at a time $t$ and at a site $(\lambda, \phi)$, where $\lambda$ is the latitude and $\phi$ is the longitude. If $(I, D)$ is a well averaged direction, so that we may take it to be the direction of the axial dipole field, the position of the corresponding paleomagnetic pole $(\Lambda, \Phi)$ can be determined using the trigonometric formulas of spherical triangle:

$$
\begin{gathered}
\sin \Lambda=\sin \lambda \cos \theta+\cos \lambda \sin \theta \cos D, \\
\cos (\Phi-\phi)=\frac{\cos \theta-\sin \lambda \sin \Lambda}{\cos \lambda \cos \Lambda},
\end{gathered}
$$

where $\theta$ is the magnetic colatitude

$$
\cot \theta=\frac{1}{2} \tan I
$$

Equation (2) is a modified form of cosine formula of spherical triangles which replaces the usual form quoted in most text books (e.g., McElhinny, 1973, p. 25) derived from the sine formula. This form is superior to the more conventional one since the longitude of the pole $\Phi$ can be unambiguously determined by taking the principal value of $\arcsin (0-\pi)$ of the right hand side of (2) and assigning the sign as the same as that of $\sin D$, e.g.,

$$
\Phi-\phi \geq 0 \text { for } 0 \leq D<\pi, \quad \Phi-\phi<0 \text { for } \pi \leq D<2 \pi .
$$

When the location of the site $(\lambda, \phi)$ is given, the paleomagnetic direction $(I, D)$ uniquely determines the pole position $(\Lambda, \Phi)$. On the other hand, when $(I, D)$ and $(\Lambda, \Phi)$ are known, they do not determine the site location $(\lambda, \phi)$ uniquely (cf. Fig. 1c). This is the fundamental non-uniqueness in paleomagnetic data mentioned earlier.

\subsection{Degree of freedom}

Let us consider a case where we have paleomagnetic data for $N$ time steps from $M$ blocks. The data may be in the form of magnetic directions or poles calculated by the dipole formula (1)-(3). The model we are seeking is the positions of the blocks in the past; i.e., $M \times N$ sets of triple numbers $\left(\lambda_{i j}, \phi_{i j}, \psi_{i j}\right)$, which represent the latitude, longitude, and orientation angle of $i$ th block at $j$ th time. (Throughout this paper, block number and time are designated by the suffixes $i$ and $j$, respectively.) There are $3 M N$ or $3 M(N-1)$ model parameters depending on either the model describes the absolute or relative movements. Clearly, there are too many degrees of freedom in the model since the number of paleomagnetic data is only $2 M N$. Therefore, we have to reduce the number of parameters in order to obtain meaningful models. 
For this purpose, we shall adopt a formulation in which the model is composed of the positions of the common poles at various time steps $\left(\Lambda_{j}, \Phi_{j}\right.$, for $\left.j=1, \ldots, M\right)$, and that the positions of the blocks at each time step $\left(\lambda_{i j}, \phi_{i j}, i=1, \ldots, N\right)$ are uniquely determined by a method, which will be defined later, from the pole position data $\left(\Lambda_{j}, \Phi_{j}\right)$, paleomagnetic direction $\left(I_{i j}, D_{i j}\right)$, and the position of that block at the adjacent time step $\left(\lambda_{i, j-1}, \phi_{i, j-1}\right)$. By this procedure, the number of model parameters is reduced to $2 M$, if the model does not allow any errors. If error is permitted, the number of model parameters becomes $2 M+M N$, where $M N$ corresponds to the freedom in choosing the angle of discrepancy $\Delta_{i j}$ between the common pole $\left(\Lambda_{j}, \Phi_{j}\right)$ and a pole calculated for a block at the same age $\left(\Lambda_{i j}, \Phi_{i j}\right)$ :

$$
\cos \Delta_{i j}=\sin \Lambda_{i j} \sin \Lambda_{j}+\cos \Lambda_{i j} \cos \Lambda_{j} \cos \left(\Phi_{i j}-\Phi_{j}\right)
$$

The number of freedom in this case is rather large, but it is permissible since it is less than the number of data, and also because $\Delta_{i j}$ can take only a limited range of values.

\subsection{Basic assumptions and choice of the penalty function}

Geophysical inverse problems can in general be formulated as a variational problem in which a function of the form

$$
S=S_{1}+\gamma S_{2}
$$

is minimized. Here, $S_{1}$ is the misfit between the observed and predicted values (e.g., the rootmean-square difference between the model and the observed data), $S_{2}$ is a regularization (or penalty) function which should take as small a value as possible because of some physical reasons, and $\gamma$ is a (positive) damping constant representing the relative weight of the two. For the misfit, a reasonable choice is to take the weighted mean square value of $\Delta_{i j}$ as defined above.

The choice and evaluation of the penalty function is a more complicated matter. To select a reasonable penalty function, we have to examine the dynamical aspects of plate motions and related phenomena. First, we note that the block motions are so slow, of the order of 100 $\mathrm{mm} /$ year at most, that inertial terms can safely be ignored. This means that the velocity or the displacement of a block at a particular time step can be determined independently disregarding the history of the former movements. Second, it can be concluded from a similar reasoning that the direction of time is not significant in the present problem. In other words, we can start the inversion from the most recent epoch and continue to go back in time one step at a time.

As stated earlier, paleomagnetic data can be explained by a combination of block (plate) motion and true polar wander. However, estimation of the true polar wander is a quite difficult problem (see Gordon, 1987). Moreover, there are cases in paleomagnetic reconstructions in which determination of true polar wander is not at all possible because the blocks involved cover only small part of the earth's surface (e.g., the two-plate problem below). Consequently, we will assume that polar wander can occur freely (at no cost). This will constitute the third basic assumption in our modeling.

Analysis of the present day plate motions demonstrated that the main driving force of plate motions is the "slab pull" and the largest force opposing to it is the "slab resistance" (Forsyth and Uyeda, 1975). In paleomagnetic reconstructions, however, the existence or non-existence of a slab attached to a block is usually not apparent, so it is perhaps permitted to assume that the "continental drag" type resistance is the main force opposing against the motion of a block in our problem. The total work needed to be done against the drag force can be evaluated by considering the area swept by a block between two consecutive time steps and summing this quantity for all the blocks and for all the time steps in question. We shall call this quantity the "total slip" and use this as the penalty function $S_{2}$ of our inverse problem. 


\subsection{Movement with least slip}

In order to reduce the number of model parameters, we presumed earlier that the positions of the blocks at individual time steps will be automatically determined by some method. As we selected the total slip as the penalty function, it is natural to require that each movement of a block also satisfies the minimum slip condition. Here, we will show that such an "automatic" determination of the movement is indeed possible.

Consider the movement of a block from one point to another lying on the surface of a sphere of unit radius (Fig. 2). For simplicity, we will assume that the block can be approximated as a circular spherical cap and take the coordinate of its center to define the position of the block.

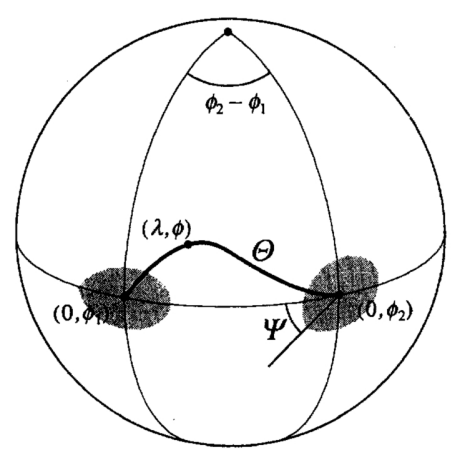

(a)

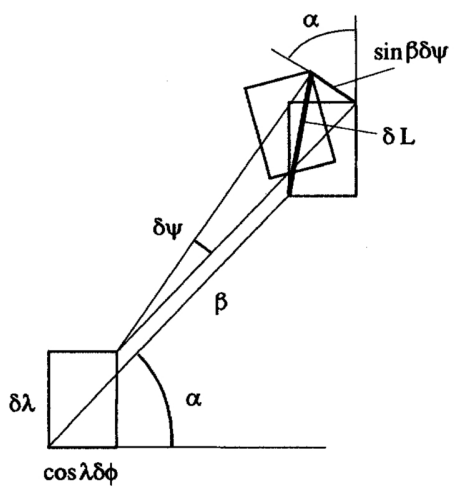

(b)

Fig. 2. Movement of a block on a sphere. (a) The start and end points are placed on the equator separated by a distance $\Theta$. The block (shaded) must also rotate by an amount $\Psi$ while moving. (b) The infinitesimal slip $d \ell$ of a small area $d A$ is caused by translation in latitudinal and longitudinal directions $(d \lambda, \cos \lambda d \phi)$ and by rotation $(d \psi)$.

With appropriate selection of the coordinate axes, we can make the start and end points to lie on the equator of the sphere. Let the coordinates of these two points be $\left(0, \phi_{1}\right)$ and $\left(0, \phi_{2}\right)$, where $\phi_{1}=0$ and $\phi_{2}=\Theta$, and assume that the block is also required to change its azimuth with respect to the equator by an angle $\Psi$ while moving between these two points (Fig. 2a). Now, take a small area $d A(=\sin \beta \sin \alpha d \alpha d \beta)$ which lies in an azimuthal direction $\alpha$ and at an angular distance $\beta$ from the center of the block (Fig. 2b). If the block moves $d \lambda$ and $\cos \lambda d \phi$ in latitudinal and longitudinal directions and rotate around the center of the block by an amount $d \psi$ between times $t$ and $t+d t$, the distance $d \ell$ by which the small area $d A$ sweeps the spherical surface can be written as

$$
(d \ell)^{2}=(\cos \lambda d \phi-\sin \beta \sin \alpha d \psi)^{2}+(d \lambda+\sin \beta \cos \alpha d \psi)^{2} .
$$

If we average this expression over the surface of the total area of the block, the terms linear in $\sin \alpha$ or $\cos \alpha$ will be eliminated and we obtain

$$
\overline{(d \ell)^{2}}=\cos ^{2} \lambda(d \phi)^{2}+(d \lambda)^{2}+(C / A)(d \psi)^{2},
$$

where

$$
A=\int d A, \quad C=\int \sin ^{2} \beta d A,
$$

are the total area and "moment of inertia" of the block, respectively. 
As it is obvious that $\phi$ increases monotonically with time in models which satisfies the minimum slip condition, we can replace the independent variable $t$ by $\phi$ without loss of generality. From (8), the distance swept by the small area $d A$ can be written as $d \ell=L d \phi$, where

$$
L(\phi, \lambda, \dot{\lambda}, \psi, \dot{\psi})=\left[\cos ^{2} \lambda+\left(\frac{d \lambda}{d \phi}\right)^{2}+\frac{C}{A}\left(\frac{d \psi}{d \phi}\right)^{2}\right]^{1 / 2}
$$

and dots represent the derivatives with respect to $\phi$. The product $L A d \phi$ gives the total amount of slip the block experiences within a short time $d t$. Our variational problem is now formulated as seeking a movement which minimizes the total slip of the block expressed as

$$
W=\int_{\phi_{1}}^{\phi_{2}} A L(\phi, \lambda, \dot{\lambda}, \psi, \dot{\psi}) d \phi
$$

with the boundary conditions

$$
\lambda=0, \quad \psi=0 \quad \text { at } \quad \phi=\phi_{1}(=0), \quad \lambda=0, \quad \psi=\Psi \quad \text { at } \quad \phi=\phi_{2}(=\Theta) .
$$

The Euler equations for this problem are

$$
\begin{aligned}
& L^{3}\left(\frac{\partial L}{\partial \lambda}-\frac{d}{d \phi} \frac{\partial L}{\partial \dot{\lambda}}\right)=-\sin \lambda \cos \lambda\left[\cos ^{2} \lambda+2\left(\frac{d \lambda}{d \phi}\right)^{2}+\frac{C}{A}\left(\frac{d \psi}{d \phi}\right)^{2}\right] \\
&-\frac{d^{2} \lambda}{d \phi^{2}}\left[\cos ^{2} \lambda+\frac{C}{A}\left(\frac{d \psi}{d \phi}\right)^{2}\right]+\frac{C}{A} \frac{d \lambda}{d \phi} \frac{d \psi}{d \phi} \frac{d^{2} \psi}{d \phi^{2}} \\
&= 0, \\
& L^{3} \frac{d}{d \phi} \frac{\partial L}{\partial \dot{\psi}}=\frac{C}{A} \frac{d^{2} \psi}{d \phi^{2}}\left[\cos ^{2} \lambda+\left(\frac{d \lambda}{d \phi}\right)^{2}\right]+\frac{C}{A}\left(\sin \lambda \cos \lambda-\frac{d^{2} \lambda}{d \phi^{2}}\right) \frac{d \lambda}{d \phi} \frac{d \psi}{d \phi}=0
\end{aligned}
$$

By eliminating $d^{2} \psi / d \phi^{2}$ from these two equations, we obtain

$$
\left[\cos ^{2} \lambda+\left(\frac{d \lambda}{d \phi}\right)^{2}+\frac{C}{A}\left(\frac{d \psi}{d \phi}\right)^{2}\right]\left[\cos ^{2} \lambda \frac{d^{2} \lambda}{d \phi^{2}}+\sin \lambda \cos \lambda\left\{\cos ^{2} \lambda+2\left(\frac{d \lambda}{d \phi}\right)^{2}\right\}\right]=0
$$

The first square bracket is equal to $L^{2}$ and positive definite. This term appeared here because we defined the Euler equations in a form multiplied by $L^{3}$. The expression in the second square bracket can be reduced to the form

$$
\frac{d^{2}}{d \phi^{2}} \tan \lambda+\tan \lambda=0
$$

which has the general solution

$$
\tan \lambda=B \cos \phi+D \sin \phi
$$

From the boundary condition at $\phi=\phi_{1}, B$ must be zero. From the condition at $\phi=\phi_{2}, D$ must also be zero unless $\Theta=\pi$. In the case that $B=D=0$, the path is along the equator and thus along the great circle connecting the two end points. When $\Theta=\pi, D$ is arbitrary and $\tan \lambda=D \sin \phi$ defines a family of great circle paths connecting the antipodal points. In both cases, $d^{2} \psi / d \phi^{2}=0$ from (14) and (15), so that the block moves along the great circle path accompanied with a rotation at a constant rate around its center. 
Finally, the amount of the slip by this movement of the block is

$$
W=\int_{\phi_{1}}^{\phi_{2}} A\left[1+\frac{C}{A}\left(\frac{d \psi}{d \phi}\right)^{2}\right]^{1 / 2} d \phi=A\left[\Theta^{2}+\frac{C}{A} \Psi^{2}\right]^{1 / 2}
$$

Thus in our approximation, the amount of slip is easily obtained when the initial and final positions of the block as well as the amount of rotation between these two positions are given.

\subsection{Destination of the block movement}

Let us assume that a polar wander path $\left(\Lambda_{j}, \Phi_{j}, j=1, \ldots, M\right)$ common to all blocks is defined. By the third basic assumption, we can determine the position of a block in the most recent past using the paleomagnetic data of that age and the present position of the block. When this is done, we can repeat the same procedure to obtain the position one step further back in time, until all the paleoposition of the block is determined. But how the position can actually be determined?

In Fig. 3, the block is situated at $\left(\lambda_{1}, \phi_{1}\right)$ and the pole of the next time step is at $\left(\Lambda_{2}, \Phi_{2}\right)$. At a first glance, it may appear that the block moves in the next time step to $\left(\lambda^{\prime}, \phi^{\prime}\right)$ which lies on the great circle connecting $\left(\lambda_{1}, \phi_{1}\right)$ and the pole. This is not always the case, however. Because there are two ingredients in the total slip (18), namely the translation $\Theta$ and rotation $\Psi$, the point satisfying both the dipole formula and the minimum slip condition is displaced from the point on the great circle by an angle $\delta$ to $\left(\lambda_{2}, \phi_{2}\right)$. This position can be obtained by searching the minimum of (18) while satisfying the dipole formula (3).

To determine the destination $\left(\lambda_{2}, \phi_{2}\right)$, we need to know the change of total slip accompanied

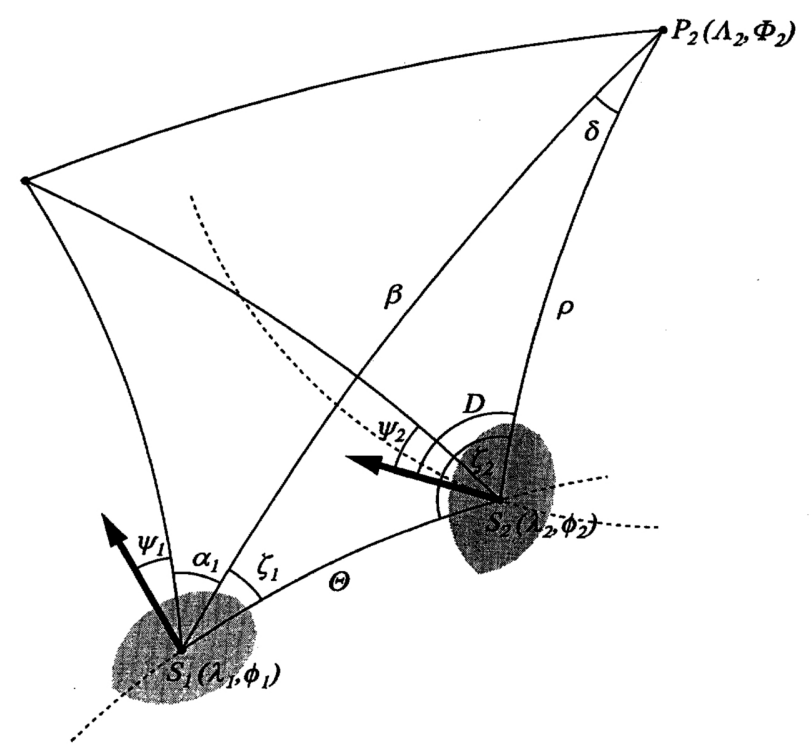

Fig. 3. When initial position of the block $\left(\lambda_{1}, \phi_{1}\right)$ and the final pole position $\left(\Lambda_{2}, \Phi_{2}\right)$ are given, the destination of the movement $\left(\lambda_{2}, \phi_{2}\right)$ is determined by the balance between the translation $\Theta$ and rotation $\Psi$ to make the amount (18) the minimum. 
with the change in the azimuthal angle $\delta$. From Fig. 3, it can be seen that

$$
\begin{aligned}
& \cos \zeta_{1}=\frac{\cos \rho-\cos \beta \cos \Theta}{\sin \beta \sin \Theta}, \quad \sin \zeta_{1}=\frac{\sin \rho \sin \delta}{\sin \Theta}, \\
& \cos \zeta_{2}=\frac{\cos \beta-\cos \rho \cos \Theta}{\sin \rho \sin \Theta}, \quad \sin \zeta_{2}=\frac{\sin \beta \sin \delta}{\sin \Theta},
\end{aligned}
$$

and

$$
\cos \Theta=\cos \rho \cos \beta+\sin \rho \sin \beta \cos \delta .
$$

Since the rotation $\Psi$ satisfies the relation $\Psi=\left(\pi-\zeta_{2}+D\right)-\left(\zeta_{1}+\alpha_{1}+\psi_{1}\right)$, it can be shown that

$$
\cos (\Psi-\chi)=-\cos \left(\zeta_{1}+\zeta_{2}\right)=\frac{\sin \rho \sin \beta+(1+\cos \rho \cos \beta) \cos \delta}{1+\cos \Theta}
$$

and

$$
\sin (\Psi-\chi)=\sin \left(\zeta_{1}+\zeta_{2}\right)=\frac{(\cos \rho+\cos \beta) \sin \delta}{1+\cos \Theta}
$$

where $\chi\left(=D-\alpha_{1}-\psi_{1}\right)$ as well as $\beta$ and $\rho$ are constant angles independent of $\delta$. Differentiation of $(22)$ by $\delta$ yields

$$
\frac{\partial}{\partial \delta} \sin (\Psi-\chi)=\frac{(\cos \rho+\cos \beta) \cos (\Psi-\chi)}{1+\cos \Theta} .
$$

Combining these, the $\delta$-derivative of the quantity in the square bracket on the right hand side of Eq. (18) can be evaluated as

$$
\begin{aligned}
\frac{\partial}{\partial \delta}\left[\Theta^{2}+\frac{C}{A} \Psi^{2}\right] & =-\frac{\Theta}{\sin \Theta} \frac{\partial}{\partial \delta} \cos \Theta+\frac{C}{A} \frac{\Psi}{\cos (\Psi-\chi)} \frac{\partial}{\partial \delta} \sin (\Psi-\chi) \\
& =-\frac{\Theta \sin \rho \sin \beta \sin \delta}{\sin \Theta}+\frac{C}{A} \frac{\Psi(\cos \rho+\cos \beta)}{1+\cos \Theta} .
\end{aligned}
$$

Equating this derivative to zero, the value of $\delta$ which minimizes the slip can be obtained as

$$
\sin \delta=\frac{C}{A} \frac{\cos \rho+\cos \beta}{\sin \rho \sin \beta} \frac{\Psi \sin \Theta}{\Theta(1+\cos \Theta)} .
$$

On the right hand side of the above formula, $\Psi$ and $\Theta$ depend on the value of $\delta$, but their change with $\delta$ is quite slow. So the optimum value of $\delta$ can be determined easily by using the above formula iteratively. This concludes the method of obtaining the destination of block motion.

\subsection{Method of calculation}

So far, we concentrated our effort for obtaining the model and for defining the penalty function $S_{2}$. Now we consider the overall scheme of the inversion. The model to be evaluated will be given as the common polar wander path. For a model the penalty $S_{2}$ will be calculated as

$$
S_{2}=\sum_{i=1}^{N} \sum_{j=1}^{M} A_{i}\left[\Theta_{i j}^{2}+\frac{C_{i}}{A_{i}} \Psi_{i j}^{2}\right]^{1 / 2}
$$

where $A_{i}$ and $C_{i}$ are the area and moment of inertia of the $i$ th block, and $\Theta_{i j}$ and $\Psi_{i j}$ are the angular distance and rotation angle in the movement of the $i$ th block between the time step $j-1$ and the time step $j$, which are determined by the scheme explained above.

For the misfit function, we take note of the following fact. The uncertainty in paleomagnetic data are usually given by the precision parameter $\kappa$ or $\alpha_{95}$, the semiangle of cone of confidence 
at $95 \%$ level. It is well known that $2 / \kappa$ closely approximates the angular variance $\sigma^{2}$ when the samples are taken from a Fisher distribution (Cox, 1970). Consider a statistic $\Delta_{i j}^{2} /\left(2 / \kappa_{j}\right)$ where $\Delta_{i j}$ is defined in (5) and represents the angular distance between the common pole of $j$ th time step and that calculated from the $i$ th block, and $\kappa_{j}$ is the precision parameter for that pole. It should approximate a two-dimensional normal distribution with variance 1 . Therefore, it is appropriate to assume that the sum of this statistic is about $M N$. This can be accommodated by requiring the misfit function to take a value expected from these uncertainties (Shure et al., 1982; Parker and Shure, 1982). In the present case, we can take $S_{1}$ as

$$
S_{1}=\sum_{i=1}^{N} \sum_{j=1}^{M} \frac{\left(\cos ^{-1}\left[\sin \Lambda_{j} \sin \Lambda_{i j}+\cos \Lambda_{j} \cos \Lambda_{i j} \cos \left(\Phi_{j}-\Phi_{i j}\right)\right]\right)^{2}}{2 / \kappa_{j}}-M N .
$$

With the inclusion of misfit in the evaluation, it is also necessary to determine the magnitude of the trade-off parameter $\gamma$ in (5), but we will postpone the discussion about its value to a later section.

The inclusion of misfit in the inversion scheme greatly increases the degree of freedom of the models. As we will show, this inverse problem is quite nonlinear and increase in the dimension of the model space makes the matter worse. Consequently, it is appropriate to ignore the misfit at first and obtain a model which only satisfies the condition that $S_{2}=\min$. Subsequently, misfit between the common polar wander path and individual polar wander path will be introduced as perturbation. This procedure is reasonable because of the high nonuniqueness of paleomagnetic data. Unlike most geophysical inverse problems, it is quite easy to make the misfit zero in the paleomagnetic case; it only needs to place the block on a small circle centered around the pole $(\Lambda, \Phi)$ with radius $\theta$ defined by (3). This will also help in concentrating our attention to the important question of the behavior of the penalty function $S_{2}$.

With these considerations, the inversion can be carried out by the following scheme:

(1) Assume a starting polar wander path $\left(\Lambda_{j}, \Phi_{j}\right), j=1, \ldots, M$. In all the models, the pole position at time $0\left(\Lambda_{0}, \Phi_{0}\right)$ should coincide with the north pole.

(2) Calculate the positions of $N$ blocks at each time step starting from $j=1$, and going backwards in time, so as to satisfy the minimum slip condition in each time interval.

(3) Calculate the total slip $S_{2}$ for this model.

(4) If the total slip converged satisfactorily, go to step 6.

(5) Search for a better model with smaller total slip. A usual procedure is to calculate the partial derivatives $\partial L / \partial \Lambda_{j}$ and $\partial L / \cos \Lambda_{j} \partial \Phi_{j}$ for the model $\left(\Lambda_{j}, \Phi_{j}\right)$, and replace the model by $\Lambda_{j}+p d \Lambda_{j}$ and by $\Phi_{j}+p d \Phi_{j}$, where

$$
d \Lambda_{j}=-\frac{\partial L}{\partial \Lambda_{j}} /\left[\sum_{k}\left(\frac{\partial L}{\partial \Lambda_{k}}\right)^{2}\right]^{1 / 2}
$$

and similarly for $d \Phi_{j}$. The parameter $p$ is changed to search for the minimum in this steepest gradient direction. Go to step 2.

(6) Incorporate the misfit $S_{1}$, and determine the model which has appropriate errors and which gives the overall minimum of the sum $S_{1}+\gamma S_{2}$.

(7) Stop calculation if the convergence is satisfactory. Otherwise, repeat the above procedures from step 6.

\section{Application to a Simple Two-Plate Problem}

With the scheme presented above, we can now proceed to solve actual problems. For the sake of simplicity and to gain insight in the characteristics of this inversion, we applied the method 
to a simple two-block problem: the reconstruction of Europe and North America. Pole positions compiled by McElhinny (1973) was used as the paleomagnetic data (Table 1). In this problem, the number of blocks $(N)$ is 2 and the number of time steps $(M)$ is 9 . Europe was approximated by a spherical cap of radius $15^{\circ}$ centered at $\left(55^{\circ} \mathrm{N}, 20^{\circ} \mathrm{E}\right)$, while North America was assumed to be centered at $45^{\circ} \mathrm{N}, 100^{\circ} \mathrm{W}$ with a radius of $25^{\circ}$.

For this classical example of continental drift, Bullard et al. (1965) obtained an Euler pole $\left(88.5^{\circ} \mathrm{N}, 27.7^{\circ} \mathrm{W}\right)$ and a rotation angle $\left(38.0^{\circ}\right)$ corresponding to the reconstruction at Jurassic age, based on the fit of the $500-\mathrm{fm}$ isobath (the Bullard fit). McElhinny (1973) showed that this Euler rotation quite well explains the difference between the APWPs of North America and Europe (Fig. 4). The Bullard fit provides a useful check for successful reconstructions.

Table 1. Paleomagnetic Poles for Europe and North America after McElhinny (1973).

\begin{tabular}{|c|c|c|c|c|c|}
\hline \multirow[b]{2}{*}{ step } & \multirow[b]{2}{*}{ age } & \multicolumn{2}{|c|}{ Europe } & \multicolumn{2}{|c|}{ North Amecica } \\
\hline & & $\Lambda\left({ }^{\circ} \mathrm{N}\right)$ & $\Phi\left({ }^{\circ} \mathrm{E}\right)$ & $\Lambda\left({ }^{\circ} \mathrm{N}\right)$ & $\Phi\left({ }^{\circ} \mathrm{E}\right)$ \\
\hline 1 & Upper Tertiary & 79 & 183 & 87 & 140 \\
\hline 2 & Lower Tertiary & 75 & 164 & 85 & 197 \\
\hline 3 & Cretaceous & 80 & 162 & 64 & 187 \\
\hline 4 & Jurassic & 65 & 138 & 76 & 142 \\
\hline 5 & Triassic & 50 & 150 & 62 & 100.5 \\
\hline 6 & Permian & 44 & 161 & 46 & 117 \\
\hline 7 & Carboniferous & 37 & 167 & 37 & 126 \\
\hline 8 & Silurian-Devonian & 27 & 162 & 29 & 123 \\
\hline 9 & Cambrian & 8 & 189 & 7 & 140 \\
\hline
\end{tabular}

\subsection{Steepest gradient method}

We found that the least-squares procedure applied to this inversion highly nonlinear, and that the results of calculations depend strongly on the starting model. Therefore, calculation may stop when one of the local minima is reached. Figure 5 shows examples from some of the results obtained by the use of the steepest decent method. When the calculation was started from a model with all the poles fixed at the geographic north pole $\left(\Lambda_{j}=90^{\circ} \mathrm{N}, \Phi_{j}=0^{\circ}, j=1, \ldots, M\right)$, the obtained positions of the continents in the past were quite different from what we expect and the amount of the total slip for the converged model is quite large (Fig. 5, left). In this model, the two continents stay near the present longitudinal bands and their movements are essentially north-south. If the mean of the APWPs of Europe and North America weighted by their respective areas is used as the initial polar wander path, the slip becomes much smaller, although the relative position is still not very similar to the Bullard fit (Fig. 5, middle). Starting from the poles fixed at $\left(30^{\circ} \mathrm{N}, 150^{\circ} \mathrm{E}\right)$ for all the time steps $(j=1, \ldots, M)$, a more reasonable reconstruction with substantial closure of the Atlantic Ocean is obtained and the amount of slip is the smallest in all the models tried (Fig. 5, right).

The final polar wander path as well as the resulting model of reconstruction depends on the starting polar wander path. To show these effects, calculations were repeated with a very large number of different starting models. In each starting model, all the poles were placed at the same place $\left(\Lambda_{j}=\Lambda, \Phi_{j}=\Phi, j=1, \ldots, M\right)$. Figure 6 shows the contour map of the total slip corresponding to these different starting models. The global minimum of the total slip is achieved by a model which at the start had the poles near $30^{\circ} \mathrm{N}, 160^{\circ} \mathrm{E}$. It can be seen that the shape of the contour is very complicated. There are a number of local minima, so that the calculation may stop by locating any one of them.

Although the starting model dependence causes serious effects, each result is well defined: 


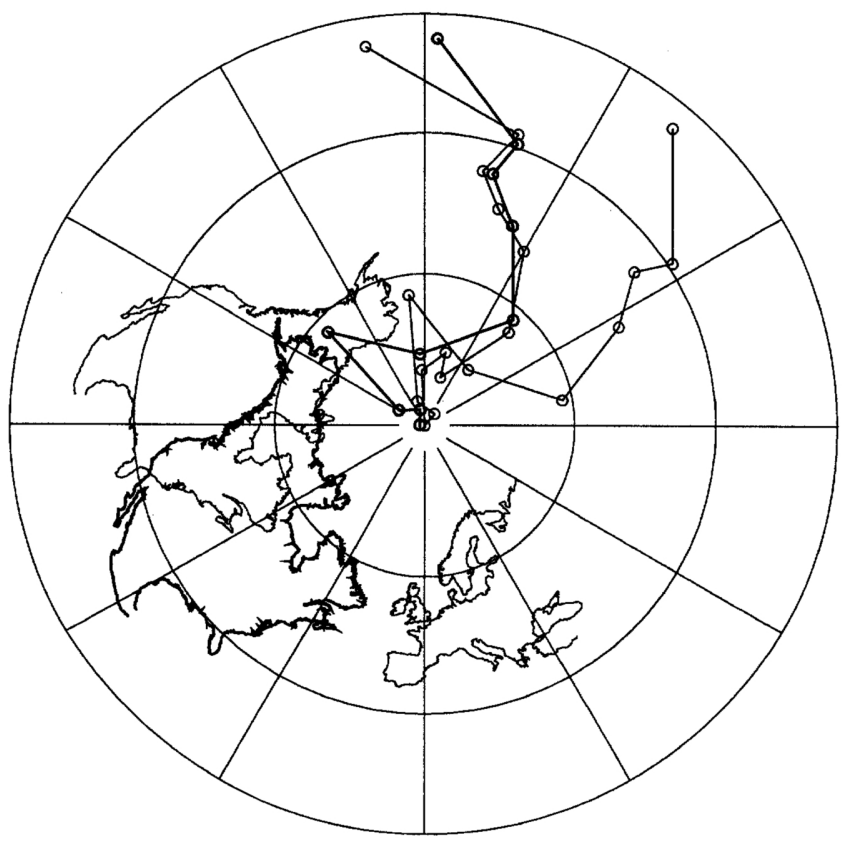

Fig. 4. Apparent polar wander path of Europe and North America (McElhinny, 1973), and position of North America when the Atlantic Ocean is closed (Bullard et al., 1965). The relocated APWP of North America (thick line) agrees well with that of Europe.

starting model I

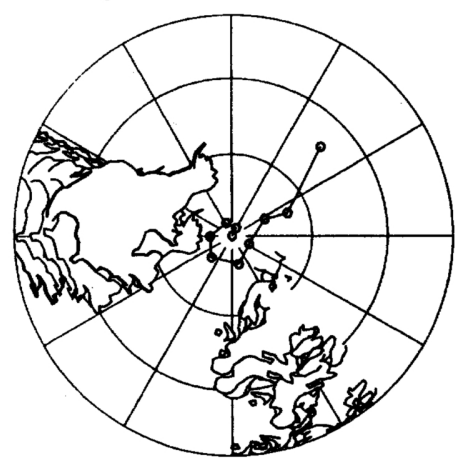

total slip $=6.60$

\section{starting model II}

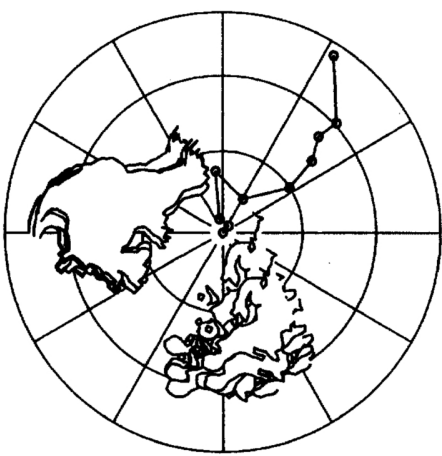

total slip $=3.57$

\section{starting model III}

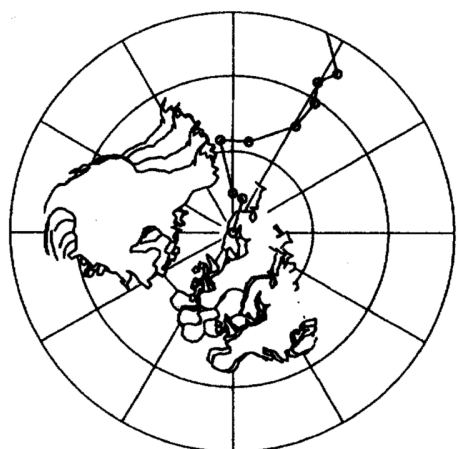

total slip $=3.35$

Fig. 5. Results of calculation by the steepest descent method shown in Lambert equal area projection. Paleomagnetic data and parameters are summarized in Table 1. Each continent is drawn from older to younger ages, with the overlapping parts of the earlier times hidden by those of the later ages. (Left) Starting poles are all at $\left(90^{\circ} \mathrm{N}, 0^{\circ}\right)$. (Middle) Starting APWP is the mean of APWPs of Europe and North America weighted by their respective areas. (Right) Starting poles are all at $\left(30^{\circ} \mathrm{N}, 160^{\circ} \mathrm{E}\right)$. 


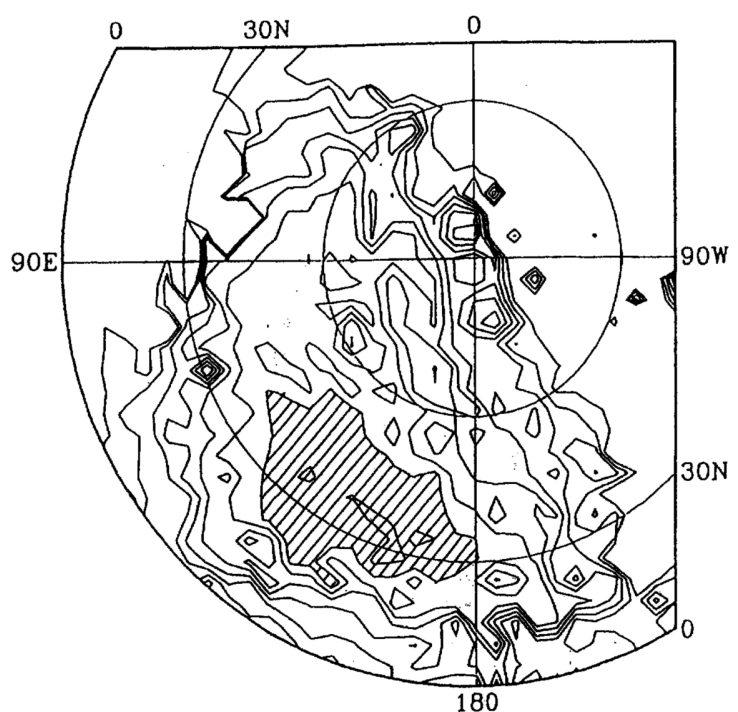

Fig. 6. Contour of the total slip for various models of reconstructions of Europe and North America. Each model starts with all the poles at the same place, and the amount of slip for the converged model is indicated at the position the poles were placed initially. The minimum of the slip is obtained by the models starting from about $\left(30^{\circ} \mathrm{N}, 160^{\circ} \mathrm{E}\right)$. Contour values are from 3.5 to 7.0 at 0.5 interval. Areas with total slip larger than 7.0 are left blank to avoid complexity. Shaded area indicates the starting models with the slip less that 4.0.

the converged models occupy well defined local minima even when the amount of slip is rather large. Each pole, except the oldest one is confined in a sharp minimum so that they are robust to small perturbations given to the polar wonder path.

The above results shows the highly nonlinear nature of the penalty function $S_{2}$, and that, to reach an optimum model, we have to search the $2 M$-dimensional model space from sufficiently large number of starting models. They also show that there may be multiple models with small difference in the values of the total slip but which differ significantly in the movement of the blocks (e.g., Fig. 5, middle and right). In the present case, it has already been known that the Atlantic Ocean was closed in Jurassic age (e.g. Bullard et al., 1965), so that Fig. 5 (right) is a better model. The fact that the model with the absolute minimum of $S_{2}$ gives the best fit with what actually occurred is quite encouraging, although the fit is still not perfect.

It can be concluded that the minimum slip model appears quite reasonable for the reconstruction of blocks in the past. From the experience above, we need a search scheme to locate the true minimum of $S_{2}$ because the maximum gradient method is quite inefficient for this purpose.

\subsection{Simplex method}

We have seen that the penalty function employed in the inverse problem of paleomagnetic reconstructions contain many local minima. The nonuniqueness in this problem is quite substantial, but it is gratifying that the actual movement of the continents is indeed very close to the optimum solutions minimizing the penalty function.

We are therefore faced with the need of a routine which not only explores the minimum of a function but also repeat this procedure until the global minimum is reached. By comparing a number of numerical methods of locating the minimum of a nonlinear function, we found that the simplex method (or downward simplex method) of Nelder and Mead (1965) is the most suitable for this problem. This method is described in the original reference as well as in Press et al. 
(1988). Briefly, the method can be described as the following.

A model having $n$ degrees of freedom can be expressed as a point in $n$-dimensional space. A simplex is a geometrical figure formed by $n+1$ non-degenerate points in this model space. The method compares the values of functions at these $n+1$ points and replaces the point with the highest value by a new one, and repeat this procedure until the volume of the simplex becomes so small that it can be judged that the function value converged to a minimum. Although this minimum may still be a local one, the process can be repeated with a different starting simplex until the global minimum is reached.

In the actual programming, we modified the routines given by Press et al. (1988) to allow for the fact that $\left(\Lambda_{j}, \Phi_{j}\right)$ spans the surface of the unit sphere and not the Euclidean two dimensional space. The final model does not depend too much on the starting model as shown in Fig. 7, where three different starting models of Fig. 5 are again used. Clearly, the final models are very similar to each other. The similarity is most striking between the APWPs shown in Fig. 7 (left and right). We conclude that we obtained a satisfactory model of reconstruction.
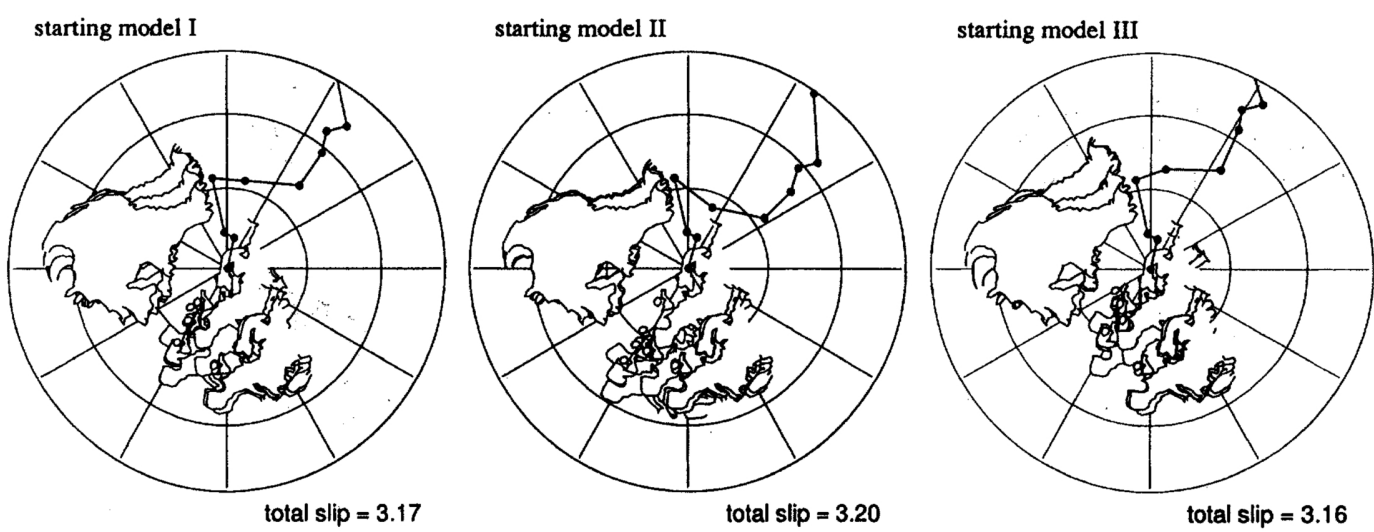

Fig. 7. Three converged models obtained by the downhill simplex method. The three panels show the results obtained from the same starting models as in Fig. 5. Remarkably similar models were obtained despite the large difference between the starting models.

\subsection{Inclusion of misfit}

Once we have the best model in terms of the total slip, we can add the misfit as perturbation. This was carried out by adding errors $\Delta_{i j}$ to individual poles and searching for the modified optimum model minimizing $S_{2}$ for a specified value of $S_{1}$. As precision parameters nor error angles were given for the paleomagnetic poles used, we chose 100 as the probable value of precision parameters $\kappa_{j}$ in Eq. (28). This is equivalent to assume that the standard angular deviation $\theta_{63}$ amounts to $8.1^{\circ}$.

Figure 8 shows the results of this search procedure, where the ordinate indicates the value of $S_{2}$ and the abscissa shows $S_{1}$ in Eq. (30) but without the term $-M N$. When misfit is allowed, the minimum of total slip is drastically decreased at first. The total slip continues to decrease, but more gradually if the allowed misfit became larger. Eventually, the rate of decrease becomes so slow that allowance of larger error does not result in a significantly better model in terms of the amount of the slip. In the present case, this transition seems to occur near $S_{1}=10$, which is slightly smaller than the optimum value of $S_{1}$ estimated from the dimension of the model $(2 M=18)$.

Choosing a damping factor $\gamma$ corresponds to selecting a different unit for either of the axes. 


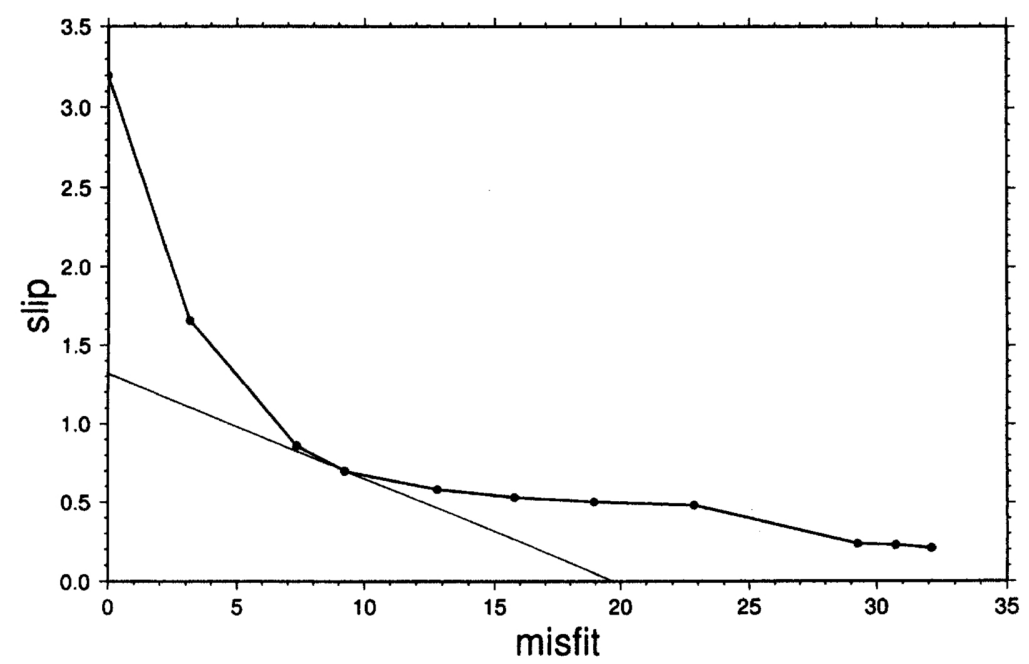

Fig. 8. Change of the total slip (ordinate) when error is allowed in the model of Fig. 7 (right). The misfit (abscissa) is measured as $\sum_{i j} \Delta_{i j}^{2} /\left(2 / \kappa_{j}\right)$, where the precision parameter was estimated to have a value of 100. The straight line tangent to the trade-off curve corresponds to the case of $\gamma=15$.
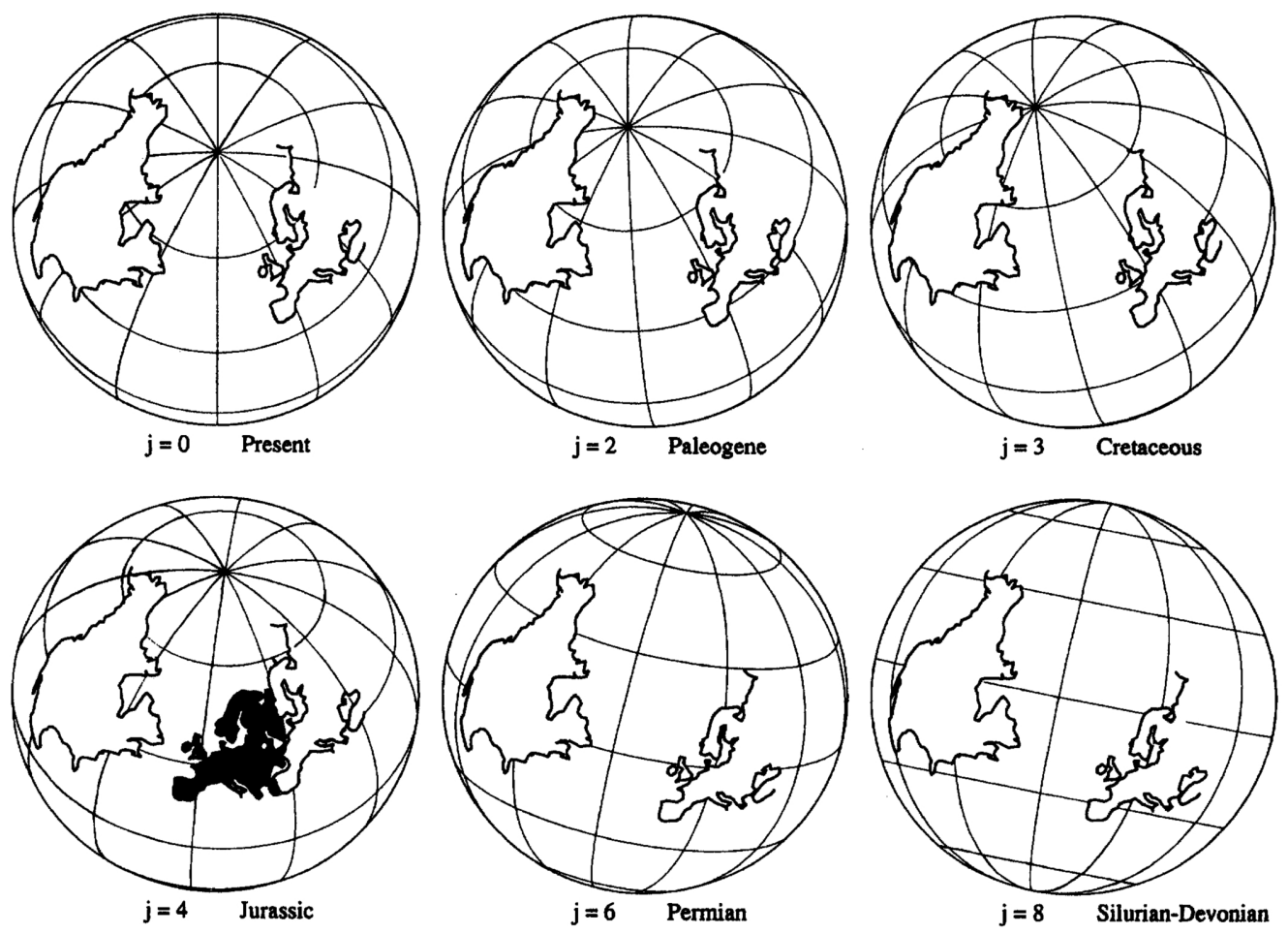

Fig. 9. The preferred model of the reconstruction of Europe and North America in the Phanerozoic, obtained by setting $\gamma=15$. All the figures are drawn in orthographic projection in which North America is fixed. Grid lines indicate latitudes and (relative) longitudes measured from the paleomagnetic pole of that time. The Bullard fit is indicated by the shaded area for the Jurassic age. 
In other word, we draw a straight line in Fig. 8 to tangent to the minimum slip-misfit curve, and choose the model which is at this tangent point. The slope of this straight line is $-1 / \gamma$. From these considerations, it appears that a damping factor of about 15 will give the most reasonable model in the present case. This will give the smallest norm or $S$ near the inflection point in Fig. 8. The fact that the value of $S_{1}$ at this point is smaller than the dimension of the model may indicate that we slightly underestimated the value of $\kappa_{j}$.

The final reconstruction incorporating the misfit is shown in Fig. 9. It can be seen that this model shows much less movement of the continents compared with Fig. 7, which did not consider the misfit. This tendency is quite natural but poses some dilemma to us. We cannot allow a large amount of misfit if we are expecting a considerable amount of block movement. Alternatively, we can only use very high quality paleomagnetic data for inversion, since the low quality data can be satisfied with a very small slip model containing large amount of misfit.

\section{Discussion}

We have succeeded in finding out an objective method which gives a reasonable reconstructions. The merit of this method lies in the fact that it is formulated as a general inverse problem. Accordingly, assessment of uniqueness or of errors in the obtained models can be done using the well established procedures for the inverse problem. However, there are still a number of problems remaining in the inversion of paleomagnetic data for the reconstruction of the position of blocks in the former ages. We will summarize some of the problems which we did not address in the present paper, and also discuss possibilities to modify the method to make it more reasonable.

It is well known that plate motion is described by an Euler rotation (e.g., Morgan, 1968), in which a block moves along a small circle path, in contradiction to the great circle path adopted in the present analysis. This difference is not serious as shown below. If a plate is to move a distance $\Theta$ with accompanied rotation $\Psi$ (Fig. 2), the angular distance $p$ to the Euler pole and the angle of rotation $Q$ can be obtained as

$$
\tan p=\frac{\sin \Theta \sin (\Psi / 2)}{1-\cos \Theta}, \quad \cos Q=\frac{\cos \Theta-\cos ^{2} p}{\sin ^{2} p} .
$$

The slip accompanied with this Euler rotation is

$$
W^{\prime}=A\left[Q^{2} \sin ^{2} p+\frac{C}{A} \Psi^{2}\right]^{1 / 2}
$$

instead of (18).

Figure 10 compares the total slip by the minimum slip method (movement on the great circle path) with that by Euler rotation for a spherical cap sith a radius of $30^{\circ}$. As seen in Fig. 10c, the difference between the two is quite small unless both $\Theta$ and $\Psi$ are very large. For smaller circular caps, the difference is even smaller. This is because the contribution to $S_{2}$ from rotation is fairly small. For a spherical cap of radius $\beta$, the area and moment of inertial are, respectively, $2 \pi(1-\cos \beta)$ and $\frac{2}{3} \pi(1-\cos \beta)^{2}(2+\cos \beta)$. The factor $C / A$ appearing in the definition of the slip is therefore only $0.015,0.059,0.128$ for the cases $\beta=10^{\circ}, 20^{\circ}, 30^{\circ}$. Thus, even if we calculate the slip using the great circle path, there is no severe conflict if we think that the movement was realized by an Euler rotation.

In this paper we inverted the Phanerozoic paleomagnetic data of Europe and North America to a common polar wander path and position of the two continent in the past, and compared this result with the Bullard fit of the North Atlantic. The results look reasonable when misfit was not allowed but the final model (containing misfit) showed very small amount of movement, because allowance for the errors tend to select models with very small slips. The use of highly accurate 


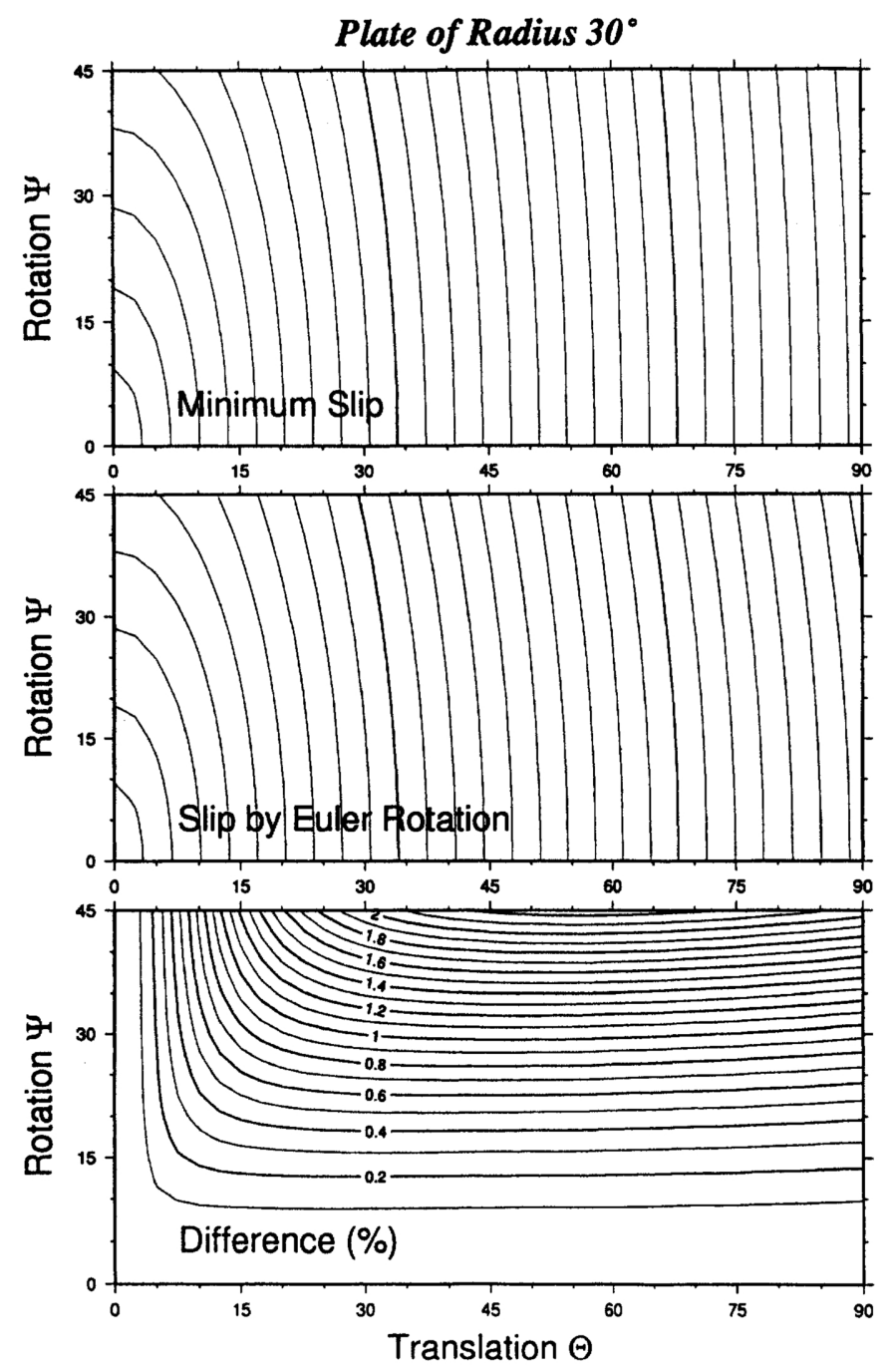

Fig. 10. The amount of slip corresponding to the minimum slip model (top), and to Euler rotation (middle), and their difference (bottom) for a spherical cap of radius $10^{\circ}$. The difference is even smaller for smaller blocks.

paleomagnetic directions will solve this problem, but in ordinary situations we may have to use error values much smaller than the actually observed ones.

Even for the no-error models (Fig. 7), we cannot deny that the inversion solution for the Jurassic is not quite the same as the Bullard fit (Fig. 4). We will meet a similar discrepancy if we compared with the more detailed sea-floor spreading data, because the latter gives essentially the Bullard fit when the North Atlantic is closed. The reason may be due to the poor quality of data especially for the Jurassic and younger ages. These poles show the largest deviation from the general trend and also when rotated back to the pre-Atlantic position, while the poles for earlier ages show very reasonable match (Fig. 4). As the present inversion scheme relies on step by step determination of the former block positions starting from the youngest age, the poor quality of data in the younger ages will adversely affect all the older reconstructions. 
Alternatively, it may be caused because it is hard to recover the movement of blocks in the longitudinal direction. The opening of the Atlantic is essentially in the east-west direction which is almost longitudinal if we note the fact that the poles were not too much displaced from the present position in these young ages. It is the ambiguity which is most difficult to resolve by paleomagnetic data, because there are always models with smaller amount of slip if we look for less longitudinal motions (cf. Fig. 1). This also casts a doubt about the appropriateness of the total slip as the penalty function for such cases. As shown in Fig. 10, this penalty is mostly determined by the amount of translation, so that the minimum slip models tend to choose movement along the meridian lines in such situations to avoid larger displacements (cf. Figs. 1(b) and $1(\mathrm{c})$ ). Similar situation can be recognized in the movement of Europe and North America, which resulted in a wider separation of the two continents compared with the Bullard fit (Fig. 9). Thus the minimum slip criterion may lead to a biased motion of blocks.

Another possibility is that the penalty function we used is intrinsically not suitable for describing the plate motion, not only for the special cases discussed above but for more general class of motions. As we said earlier, we neglect the relative importance of the plate driving forces. The real situation may be better described, for instance, by penalty function which has anisotropy depending on the direction of motions or which depends on the former position of trenches. However, for the reasons discussed earlier, the use of the penalty of the present form is perhaps the best choice for the older ages if we try to be objective in reconstruction.

In a more realistic applications, reconstructions could have been started from the Bullard fit in the Jurassic, because the positions in the later ages can be taken as precisely known thanks to the magnetic lineations in the Atlantic Ocean. This generally applies to other areas where oceanic magnetic anomalies have been successfully identified. There will be no gain of information using paleomagnetic inversion if sea floor spreading can be traced by magnetic anomalies. Thus the principal use of the paleomagnetic data is for the ages older than about 200 million years.

Although we showed a workable scheme for paleomagnetic inversion, it must be admitted that even the proper form of the penalty function is still open for discussion. Throughout this paper, we used minimum slip criterion for the penalty function. There are additional problems involved in other assumptions. For instance, we allowed the blocks to change the speed and direction of the movement freely, and we also allowed the common polar wander path to change freely. These assumptions may be subject to criticism. However, since the formulation presented in this paper is quite general and is valid, in the sense of geophysical inversion, regardless of the particular form of the penalty function, we can modify $S_{2}$ to a more realistic form and still apply the same procedures to invert the data.

There are other possible application of paleomagnetic inversion, one of which is the separation of true polar wander (e.g., Gordon, 1987). If the paleomagnetic data from all the major blocks of the world are inverted, the resulting common polar wander path may give some indication of the true polar wander. This deduction needs some reservation since the blocks included will necessarily be continental, so that contribution from the oceanic areas will be neglected. Still, it is worth trying and we are currently investigating the possibility of obtaining true polar wander from paleomagnetic inversion.

\section{Conclusions}

We have formulated paleomagnetic reconstruction as an inverse problem in which the model consists of a common polar wander path. In the inversion, first the model which gives the minimum amount of slip of the block is obtained. Errors in the position of paleomagnetic poles were then allowed and the optimum model was determined based on the combined amount of misfit and slip. For obtaining the minimum slip model, some mathematical tools are necessary, and we developed such tools to make the search possible. 
The movement which gives the minimum slip for a given pair of starting and ending positions and orientations of a block is shown to be the simple translation along the great circle path accompanied with the uniform rotation. By taking minimum slip movement for each block, complete drift of blocks (continents) can be specified if the common polar wander path is given. It was found that the total slip for all the blocks and all the ages is a very complex function and that it takes a large number of local minima, which necessitates a very extensive search to locate the global minimum in the model space. The simplex method was found to be suitable for this search.

The obtained reconstruction for the case of the reconstruction of Europe and North America looks quite reasonable, but there was certain amount of discrepancy in comparison with the Bullard fit. This may indicate that the minimum slip criterion is too naive and that other penalty function may better describe the plate motions. However, the use of total slip is still reasonable for reconstruction in older ages because this gives the most unbiased estimate of penalty in plate motions.

We wish to thank Hidefumi Tanaka for discussion. We also thank two anonymous reviewers for constructive criticisms. Contribution 19 from Department of Earth and Planetary Sciences, Tokyo Institute of Technology.

\section{REFERENCES}

Backus, G. E. and J. F. Gilbert, Numerical applications of a formalism for geophysical inverse problems, Geophys. J. Roy. Astro. Soc., 13, 247-276, 1967.

Bullard, E., J. E. Everett, and A. G. Smith, The fit of the continents around the Atlantic, Phil. Trans. Roy. Soc. London, A258, 41-51, 1965.

Cox, A., Latitude dependence of the angular dispersion of the geomagnetic field, Geophys. J. R. Astr. Soc., 20, 253-269, 1970.

Fisher, R., Dispersion on a sphere, Proc. Roy. Soc. Lond., A217, 295-305, 1953.

Forsyth, D. W. and S. Uyeda, On the relative importance of driving forces of plate motion, Geophys. J. R. Astr. Soc., 43, 163-200, 1975.

Gordon, R. G., Polar wandering and paleomagnetism, Ann. Rev. Earth Planet. Sci., 15, 567-593, 1987.

Irving, E., R. F. Emslie, and H. Ueno, Upper Proterozoic paleomagnetic poles from Laurentia and the history of the Grenville structural province, J. Geophys. Res., 79, 5491-5502, 1974.

Jackson, D. D., Interpretation of inaccurate, insufficient and inconsistent data, Geophys. J. R. astr. Soc., 28, 97-109, 1972.

McElhinny, M. W., Paleomagnetism and Plate Tectonics, 358 pp., Cambridge Univ. Press, 1973.

Morgan, W. J., Rises, trenches, great faults, and arustal blocks, J. Geophys. Res., 73, 1959-1982, 1968.

Nelder, J. A. and R. Mead, A simplex method for function minimization, Computer J., 7, 308-313, 1965.

Parker, R. L. and L. Shure, Efficient modeling of the earth's magnetic field with harmonic spline, Geophys. Res. Lett., 9, 812-815, 1982.

Press, W. H., B. P. Flannery, S. A. Teukolsky, and W. T. Vetterling, Numerical Recipes in C-The Art of Scientific Computing, 735 pp., Cambridge Univ. Press, 1988.

Shure, L., R. L. Parker, and G. E. Backus, Harmonic splines for geomagnetic modelling, Phys. Earth Planet. Inter., 28, 215-229, 1982.

Uyeda, S., The New View of the Earth-Moving Contients and Moving Oceans, W. H. Freeman and Co., San Francisco, 1978.

Vine, F. J. and D. H. Matthews, Magnetic anomalies over oceanic ridges, Nature, 199, 947-949, 1963.

Wegener, A., Die Entstehung der Kontinente und Ozeane, 4th ed., Vieweg uns Sohn, Branschweig, 1929 (English translation: The Origin of Continents and Oceans, 246 pp., Dover, New York, 1966). 